\title{
An International Trade Negotiation Framework for e-Government ${ }^{\star}$
}

\author{
Sheng Zhang ${ }^{1}$, Fillia Makedon ${ }^{1}$, James Ford ${ }^{1}$, Calliope Sudborough ${ }^{2}$, Lin $_{\mathrm{Ai}^{3}}$, \\ Sarantos Kapidakis ${ }^{4}$, Vangelis Karkaletsis ${ }^{5}$, and Euripides Loukis ${ }^{6}$ \\ 1 The Dartmouth Experimental Visualization Laboratory (DEVLAB) \\ Department of Computer Science \\ $\{$ clap, makedon, jford \}@cs.dartmouth.edu \\ 2 kalioppi@bu.edu \\ 3 erin_ai@hotmail.com \\ 4 Ionio University, Greece \\ sarantos@ionio.gr \\ 5 NCSR-Demokritos Research Institute, Greece \\ vangelis@iit.demokritos.gr \\ 6 University of the Aegean, Greece \\ eloukis@aegean.gr
}

\begin{abstract}
International trade negotiations among national governments and organizations are usually arduous and complicated. We propose a framework that supports government to government negotiation. With this framework, governments can keep track of previous negotiations using a database of negotiation records in an electronic platform. Moreover, the framework supports searching, sharing and learning past negotiation records as well as the ability to conduct negotiations on a variety of resources, products and services.
\end{abstract}

\section{Introduction}

International trade not only brings trading countries substantial economic gains from specialization and more efficient resource allocation, but also the adoption of new technologies, leading to higher productivity [1].

Generally, the traditional notion of a negotiation can be understood as the process toward a final agreement on one or more matters of common interest to different parties. Global trade negotiations are arduous and complicated as they cover all manner of goods and services. Moreover, countries often erect trade barriers over time for a variety of reasons: to protect local industries, to protect special interests and to provide stable employment. So governments negotiating with one another must find means to reduce these barriers. Finally, as the negotiations can span many years and different governments in power, agreements and sub-agreements are often cumbersome to keep track of [2].

In this paper we describe a framework that helps automate government to government negotiations, helps decide on what negotiation conditions to use,

^ This work was supported in part by NSF ITR 0312629. 
tracks agreements in time and provides decision support. With this framework, governments and organizations can conduct and keep track of previous negotiations using a database of negotiation records in an electronic, easy to use platform. Furthermore, the framework supports searching, sharing and learning past negotiation records as well as the ability to conduct negotiations on a variety of resources, products and services. Building background knowledge about previous agreements and classifying this knowledge according to the type of trade involved, the conditions, the country, or other parameters, is valuable in promoting international trade.

\section{Negotiation Scenarios and Negotiation Protocols}

During a traditional trade negotiation process, a government usually has the following requirements. Before the negotiation, it wants to find those past negotiations related to the current negotiation from all the negotiation history it keeps locally. This is usually a manual process sensitive to human judgement as to what document is relevant and to human lapses as to being able to collect all the necessary documents, which may be distributed among different sectors, physical areas and nations. Moreover, a government also wants to see any additional related negotiation history kept by the other governments involved. This history is often in different terms and susceptible to epistemological barriers.

Once all information has been gathered, a government is interested in finding useful rules hidden in these resources to help it decide the negotiation strategy it will use. After choosing the appropriate strategy, all negotiation parties must find a common time, place and mechanism to start and conduct the negotiation. This is a costly process, both in terms of time and money. Providing a trusted electronic platform to conduct this negotiation would facilitate differences in time, language and ability to access previous conditions, and would reduce the overall cost in terms of lost time. The system as we describe below would enable all or most negotiation stages to be processed automatically and act as assistants to the people involved in a physical meeting.

Generally there are two categories of negotiations in international trade agreements among governments. In the following, we will describe them and discuss the related negotiation protocol needed in each:

The first category is bilateral negotiations. An example for this case might be when two governments are trying to lower the barriers such as tariffs or quotas on the agriculture products exported to the other country, or when they are processing a large government purchase from each other.

The traditional bilateral negotiation protocol in this application has four primitives: Call for Proposals (CFP), Proposal (or Counter-Proposal), Accept and Reject. A party sends a CFP to the other party, which announces the start of negotiation. Then, both parties continuously send proposals and counter proposals to each other until one of them accepts the offer proposed by the other or one rejects continuing with the negotiation. 
The second category is multilateral negotiations. An example of this case is when there are three or more governments that are trying to reach a trade agreement enabling free trade in the affiliated countries. This kind of negotiation is popular in World Trade Organization (WTO) negotiations [3].

Multilateral negotiations can be conducted bilaterally, which means that every two parties can conduct a bilateral negotiation and reach an agreement, then all these bilateral agreements are merged to form the group agreement. Generally, these bilateral negotiations can be conducted asynchronously. However it is necessary to conduct all the bilateral negotiations simultaneously if there is a dependence in two conditions proposed in different negotiations. For example, suppose government A wishes to ensure that its tariff on government B's electronic products is the same as its tariff on electronic products it imports from country $\mathrm{C}^{1}$. Therefore, government $\mathrm{A}$ need to make sure that the condition variable (tariff on products from $\mathrm{B}$ ) in its negotiation with government $\mathrm{B}$ is always the same with the condition variable (tariff on products from $\mathrm{C}$ ) in its negotiation with government C. Moreover, sometimes parties may want to use majority rules in multilateral negotiations, which means if most governments are not satisfied with government A's performance in negotiations, they can give a warning to urge it to make satisfactory concessions or even drive it out of the final group agreement if it still fails to make any concession. In [4], we introduce a new negotiation model for reaching a group consensus in multiparty negotiations using majority rules. This model and the traditional bilateral negotiation protocol are used in our system.

\section{Proposed Framework}

There are four components in our system: the negotiation component, the sharing component, the searching component and the learning component. The details of each component are provided later in this section. We now use a simple example to illustrate how the whole framework works.

Assume the United States government wants to reach an agreement with Germany on an automobile trade issue. To prepare for such a negotiation, the United States government searches its negotiation digital library through the searching component (see figure 1) to retrieve all those past negotiation histories which relate to the German government or which relate to the automobile market. Further, the United States wants to see whether there are any other governments that have had any negotiations conducted with Germany on automobiles in the past several years. So the government uses the sharing component and finds that France just had such a related negotiation history or behavior. However, France would like to share this negotiation information with the United States only if the United States agrees to share its experience of negotiating with China on electronic products. After a series of bargaining steps, the United States and France finally reach an agreement on how to share the information of these negotiation history.

\footnotetext{
${ }^{1}$ This is a way of expressing Most Favored Nation clause.
} 


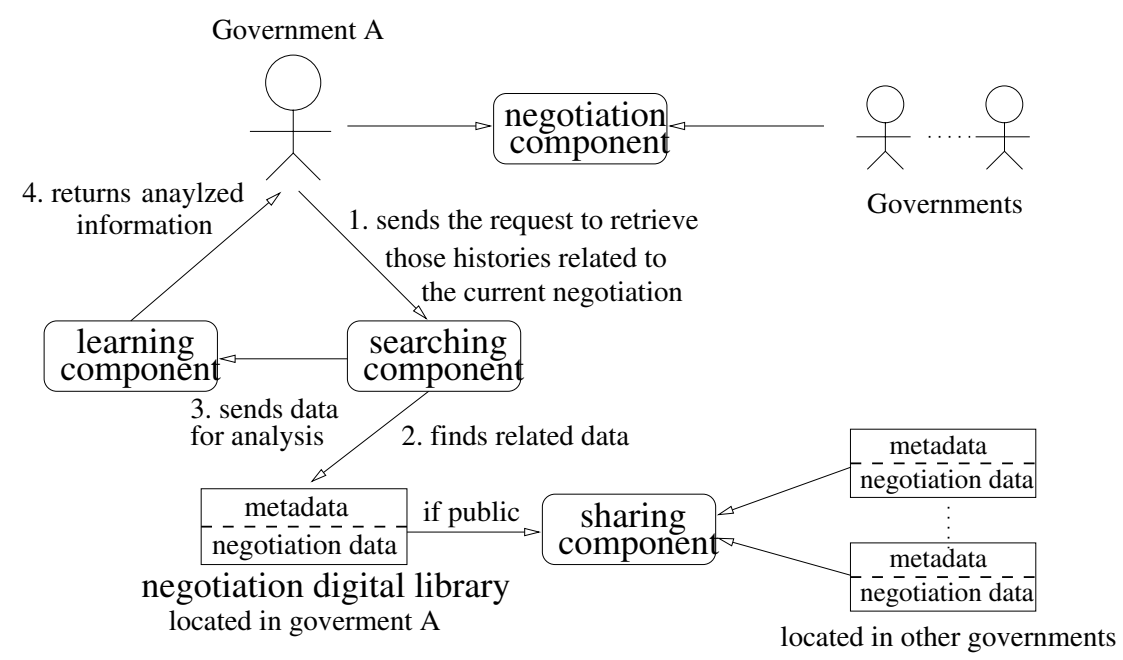

Fig. 1. The framework for trade negotiations among governments.

Once the United States has obtained all negotiation history data related to Germany and automobile products (both originally from its negotiation digital library and from other governments), it sends these data to the learning component for data analysis. The learning component helps find that using an aggressive negotiation strategy to negotiate with the German government usually gets better negotiation results. After getting this useful information from past experience, the United States government starts a formal negotiation with the German government. This negotiation can be conducted by human beings or negotiation agents, and is supported by an electronic platform through the negotiation component.

When the negotiation ends, the United States keeps all the negotiation records in its negotiation digital library. This negotiation now becomes a reference for the government when it has future negotiations with Germany on similar issues, and may also help other governments if United States shares this information with them.

\subsection{Negotiation Component (SCENS)}

Governments can choose to conduct negotiations either in person or using our negotiation component SCENS [5,6] (Secure/Semantic Content Exchange System).

SCENS is a three-layer web service based negotiation system. Layer 1 behaves as a traditional web-based negotiation support system for human beings. It provides some negotiation agents, which are actually user customizable utility functions. With this layer, we have a uniform platform for global negotiations among governments and international organizations. 
Each government or organization can register into the system and find its negotiation party (or parties). Using a friendly interface, users can enter their negotiation conditions and start a negotiation. Meanwhile, while the negotiation is going on, and if the negotiation parties want this, negotiation agents can analyze the negotiation process as negotiation assistants by suggesting a reasonable negotiation behavior (i.e., accept, decline or give a new counteroffer).

Layer 2 of SCENS provides support for complete negotiation strategy customization by users. In Layer 2, governments can have their own negotiation agents implementing any negotiation strategies. The negotiation agents, which are treated as web service consumers and run on the client side, conduct negotiation with other negotiation agents or human beings through web services.

Layer 3 of SCENS provides an open automated negotiation environment, which could allow governments to pass all the negotiation tasks to their agents and have agents complete the whole negotiation process on their behalf. In this layer, we use NOODLE (Negotiation OntOlogy Description LanguagE) [7], a language for creating negotiation ontologies (formal representations of domain knowledge) and marking up information, which allows agents to acquire knowledge about how to conduct negotiations. This knowledge includes negotiation protocols, negotiation proposals and conditions, etc. Agents communicating with Layer 3 can be used in any negotiation activities given the proper negotiation ontology. (In Layer 2, in contrast, the knowledge about negotiation rules is actually hard-coded into the agents.)

So from layer 1 to layer 3, SCENS provides various choices to government users, from the lowest level of providing an electronic platform to the highest level of supporting automated negotiations.

\subsection{Negotiation Digital Library and Sharing Component}

We envision that each government has a negotiation digital library where it maintains and updates all its past negotiation history (including those negotiations that failed to reach a deal). A metadata framework [8] is used to construct this library. Except for the original negotiation records, metadata descriptions (higher level descriptions of negotiation processes) are also stored. After each negotiation ends, metadata is extracted from the negotiation record. Then the government decides whether the original negotiation record is private or public. If it is private, then it will always be kept in the local digital library together with the corresponding metadata. If it is public, the government can add an appropriate usage policy to it. This usage policy can vary according to a variety of factors: the time of the agreement, the product, the parties involved, the sharing conditions, the laws involved and other factors. All the public metadata with a related usage policy are put onto the sharing component and can be browsed by other governments.

When another government is interested in accessing the public original negotiation records a government provides, they can negotiate about the conditions on access to this information. This type of negotiation is mainly used for sharing existing information or generated information from previous negotiations. 
It becomes a valuable commodity that can also be leveraged. In other words, a country or government that has undergone a lot of negotiations and has maintained a good record using SCENS has higher leverage than a country that just enters the game. This is used as incentive for governments to want to become part of this process. Built-in services such as these are important incentives for using SCENS and are also ways to build trust among governments. The original record is kept private until an agreement is reached on how to share the information.

\subsection{Searching Component}

The searching component helps users find those negotiation histories that relate to the current ongoing negotiation. Negotiation records are usually stored in text documents, so traditional search methods use text-based retrieval by matching the metadata with the query. These methods are relatively simple to implement and easy to use. However, there are two problems which can lead to poor retrieval accuracy. One is that different people (who may be in different countries) may use different words in metadata description, and the other is that people who know little about the domain can not always specify the most appropriate keywords for the query.

To address these two problems, we introduce an ontology based retrieval method in [9]. In ontology-based retrieval, both metadata and retrieval queries are represented by XML files based on a shared ontology that encodes semantic annotations. By extracting combined concept entity, fundamental semantic units can be extracted for both. Based on sets of combined concept entities, we compare the semantic similarity between stored data and a retrieval query. Compared with other approaches, ontology-based retrieval provides better standardization of information descriptions and potentially allows understanding of semantic content.

\subsection{Learning Component}

The learning component is in charge of finding useful rules and information in these retrieved negotiation histories. With the negotiation strategy pool framework we introduce in $[7,10]$, we can incorporate a higher level artificial intelligence into the basic traditional negotiation strategies. This higher level intelligence has the following two parts that are used to enable users to be more flexible and robust in negotiations.

A neural network helps the user choose a best fit negotiation strategy that generally works well (decided by the user satisfactory ratio) in similar negotiation environments (e.g., similar negotiation conditions, preferences and constraints). Moreover, since in each negotiation process the strategy is potentially different and is subject to revision, it is more difficult for the other agents to deduce or reason out the strategy than it would be when using, instead, a uniform negotiation strategy. 
The second part is a multidimensional data mining technique that is used to find those inter-transaction association rules hidden inside the negotiation histories. An example of such a rule is that if Germany offers a $10 \%$ concession on the tariff, one needs to give a 5\% concession as a response. Such rules are good references to the users and might be incorporated into the negotiation strategy.

\section{Conclusion}

We have presented a new e-negotiation framework for international government to government trade negotiations. This framework supports governments in (a) searching the past negotiation records, (b) finding useful information by learning from past negotiation experience, (c) sharing the negotiation records among different governments and $(\mathrm{d})$ conducting an e-negotiation or even have agents complete all negotiations.

\section{References}

1. http://www.cid.harvard.edu/cidtrade/issues/general.htm Last visited on $05 / 30 / 2004$.

2. http://www.dti.gov.uk/ewt/development.htm. Last visited on 05/30/2004.

3. http://www.wto.org. Last visited on 05/30/2004.

4. Sheng Zhang, Fillia Makedon, and Lin Ai. A model for multiparty negotiation with majority rules. To appear in International Conference on Electronic Commerce and Web Technologies (EC-WEB), 2004.

5. Song Ye, Fillia Makedon, Tilmann Steinberg, Li Shen, James Ford, Yuhang Wang, Yan Zhao, and Sarantos Kapidakis. SCENS: A system for the mediated sharing of sensitive information. In proceeding of the third ACM/IEEE-CS joint conference on Digital libraries (JCDL), pages 263-265, 2003.

6. Fillia Makedon, Song Ye, and Yan Zhao. On the design and implementation of a web-based negotiation system. In proceeding of the 9th Panhellenic Conference on Informatics (PCI), pages 46-57, 2003.

7. Fillia Makedon, Song Ye, Sheng Zhang, James Ford, Li Shen, and Sarantos Kapidakis. Data brokers: Building collections through automated negotiation. In proceeding of Hellenic Conference on Artificial Intelligence (SETN), LNCS 3025 Springer, pages 13-22, 2004.

8. Fillia Makedon, James Ford, Li Shen, Tilmann Steinberg, Andrew Saykin, Heather Wishart, and Sarantos Kapadakis. MetaDL: A digital library of metadata for sensitive or complex research data. In proceeding of European Conference on Digital Libraries (ECDL), pages 374-389, 2002.

9. Wei Zheng, Yi Ouyang, James Ford, and Fillia Makedon. Ontology-based image retrieval. In proceedings of WSEAS MMACTEE-WAMUS-NOLASC, 2003.

10. Sheng Zhang, Song Ye, Fillia Makedon, and James Ford. A hybrid negotiation strategy mechanism in an automated negotiation system. In proceeding of $A C M$ Conference on Electronic Commerce (EC), pages 256-257, 2004. 